\title{
Comparison of The Effects of Succinated Gelatine and Hydroxy Ethyl Starch (6\% 130/0.4) On Bleeding Among Cardiac Surgery Patients
}

\author{
E.Kubra Okur Kavak, MD - Prof. Bora Aykac, MD - Zeynep Celik, MD - Fusun Kaya, MD \\ Istanbul University Cardiology Institute Hospital Department of Anesthesiology
}



\section{Background}

Volume therapy is often necessary to treat hypovolemia.Especially for the cardiac surgery patients, volume deficit-replacement is more important. There are several causes of hypovolemia and should be treated according to the cause and deficit.Depends on the reason of hypovolemia we can treat it wit different fluids such as crystalloids, colloids,blood,plasma,..

All kind of surgeries even the minor one intravenous fluid replacement is necessary and done.But which solution it should be done has contraversies.Crystalloids or colloids? In our study we compared two different colloid solutions and especially their effects on bleeding for cardiac surgery patients.

\section{Objectives}

This study was to compare the effects of HES 130/0.4 and Modified Fluid Gelatine on coagulation in patients undergoing cardiac surgery.

\section{Material and methods}

: Patients were randomized into 2 groups; group I(n:30) andgroup II(n:30). Groupl contains the patients who had modified fluid gelatine infusion and group II contains the patient

swho had HES 130/0.4 infusion. Both groups had similar demographic characteristics. For both groups patients charts checked and noted preop,- postop 1sth hour and postop 1st day hgb, hct,plt, PT, aPTT, INR, BUN, Cr, ALT and AST results. These

results were analyzed SPSS statistic package. These two different colloids's effects on coagulation in cardiac surgery patients. We measured the total hgb decrease to compare. And postop 1st hour; we observed that group II patients statistically significant hgb and hct decrease. Apart from our study we also observed that group II patients had statistically significant ALT and $\mathrm{Cr}$ abnormal test results which should be discussed for an other study. After all according to 1st day lab results hgb and hct were in normal range and there were no differences between HES 130/0.4 and modified fluid gelatin on coagulation. Probably the bloodand plasma transfusion in ICU and also postop surgica Ibleeding usually occurs in the 1 st-24 hour.

\section{Results}

First 24 hours HES 130/0.4 caused coagulation impairment and significant $\mathrm{Hgb}$ and Hct decrease among postcardiac surgery patients. We also found that HES 130/0.4 caused significant ALT and $\mathrm{Cr}$ abnormality which should be further studied.

\section{Conclusions}

Although postop 1st hour HES 130/0.4 preparation caused more hgb and hct decraese in cardiac surgery patients it was temporary, safe and not dangerous amount. And for the volume replacement therapy new HES preparation was as safe as modified fluid gelatin.

Table 1/ Pre-op values
\begin{tabular}{|l|l|l|l|}
\hline & Group G (n:30) & Group V (n:30) & p \\
\hline Hb & $12.6 \pm 1.6$ & $12.4 \pm 1.4$ & 0.66 \\
\hline Hct & $38.0 \pm 4.6$ & $37.8 \pm 4.2$ & 0.86 \\
\hline Bun & $19.7 \pm 4.9$ & $26.1 \pm 16.1$ & 0.04 \\
\hline Creatinin & $0.9 \pm 0.1$ & $1.2 \pm 1.0$ & 0.1 \\
\hline ALT & $22.6 \pm 15.3$ & $27.3 \pm 17.3$ & 0.2 \\
\hline AST & $23.4 \pm 16.1$ & $26.6 \pm 19.8$ & 0.4 \\
\hline Plt & $239266.6 \pm 65437.4$ & $263733.3 \pm 88539.4$ & 0.22 \\
\hline PT & $12.4 \pm 1.1$ & $13.2 \pm 1.7$ & 0.04 \\
\hline aPTT & $31.2 \pm 7.7$ & $31.9 \pm 4.2$ & 0.9 \\
\hline INR & $0.9 \pm 0.1$ & $0.9 \pm 0.1$ & 0.8 \\
\hline
\end{tabular}

Table 2| Post-op 1st hour values

\begin{tabular}{|c|c|c|c|}
\hline & Group G (n:30) & Group V (n:30) & $p$ \\
\hline $\mathrm{Hb}$ & $10.5 \pm 1.2$ & $9.2 \pm 2.0$ & $0,004 *$ \\
\hline Hct & $31.8 \pm 4.0$ & $29.3 \pm 3.9$ & $0.01 *$ \\
\hline Plt & $158233.3 \pm 54985.6$ & $168933.3 \pm 112027.3$ & 0.6 \\
\hline PT & $14.1 \pm 1.1$ & $16.5 \pm 11.9$ & 0.2 \\
\hline aPTT & $34.0 \pm 5.7$ & $44.8 \pm 37.5$ & 0.1 \\
\hline INR & $1.1 \pm 0.1$ & $1.3 \pm 1.4$ & 0.4 \\
\hline
\end{tabular}

Table 3| Post-op day 1 values

\begin{tabular}{|l|l|l|l|}
\hline & Group G (n:30) & Group V $(\mathrm{n}: 30)$ & $p$ \\
\hline $\mathrm{Hb}$ & $10.0 \pm 1.6$ & $9.7 \pm 0.9$ & 0.3 \\
\hline Hct & $30.4 \pm 4.7$ & $30.0 \pm 3.1$ & 0.71 \\
\hline Bun & $21.4 \pm 8.8$ & $26.7 \pm 11.3$ & 0.05 \\
\hline Creatinin & $1.1 \pm 0.4$ & $1.5 \pm 0.7$ & $\mathbf{0 . 0 3 *}$ \\
\hline ALT & $24.5 \pm 15.9$ & $40.3 \pm 32.3$ & $\mathbf{0 . 0 2 *}$ \\
\hline AST & $57.5 \pm 34.1$ & $90.1 \pm 89.5$ & 0.06 \\
\hline Plt & $194666.7 \pm 87744.2$ & $197800.0 \pm 131587.7$ & 0.9 \\
\hline PT & $14.4 \pm 3.6$ & $14.3 \pm 1.7$ & 0.9 \\
\hline aPTT & $33.2 \pm 6.0$ & $36.8 \pm 14.4$ & 0.2 \\
\hline INR & $1.1 \pm 0.4$ & $1.0 \pm 1.1$ & 0.3 \\
\hline * p $<0.05($ Mean \pm SD $)$ & \multicolumn{2}{l}{} \\
\hline
\end{tabular}

